\title{
Low Cost Design and Fabrication of Microstrip Stepped-Impedance Low-Pass Filter by using FR-4 substrate
}

 \\ ${ }^{a}$ National Institute of Technology Kagawa College, 551 Kouda, Takuma-cho, Mitoyo-city 769-1101, Japan \\ *Corresponding Author: kusama@cn.kagawa-nct.ac.jp
}

\begin{abstract}
A series of process from low cost design to fabrication of a microstrip line stepped-impedance low-pass filter is examined. Regarding the decrease of the cutoff frequency that occurs in the design theory, it is found that the problem is solved by the transmission line length adjustment by referring to the commercially available microwave experiment kit using a low loss and high cost Teflon substrate. Then the same design method is applied to a low cost FR-4 substrate. It is possible to obtain the similar LPF characteristics as a commercial microwave experiment kit by selecting a characteristic impedance ratio of the stepped-impedance line appropriately combined with the $\lambda / 4$ transverse resonance technique in cutoff region. It is confirmed that the frequency characteristics of the manufactured sample agree well with the design value by the circuit simulator and the EM simulation up to $6 \mathrm{GHz}$. the manufacturing cost is reduced to about one tenth.
\end{abstract}

Keywords: microwave circuit, microwave education, CAD.

\section{Introduction}

The combination of theory, simulation, and experiment is effective for deepening all engineering disciplines, and microwave engineering is no exception. Therefore, confirmation and verification by experiments are finally required. There are many useful documents for mastering the theory of microwave engineering with the spread of the Internet, world-standard books have become readily available on an individual level ${ }^{(1,2)}$. In addition, expensive electromagnetic simulators that can be used at low cost for academics are becoming popular, and open source electromagnetic simulators have also been introduced. This trend is expected to accelerate further in the future. On the other hand for experiments, a waveguide experiment $\mathrm{kit}^{(3)}$ is well known as a microwave standard experiment kit, and recently experimental kits based on the microstrip line (MSL) are also avairable ${ }^{(4-6)}$. However, the cost of introducing microwave experimental kit are still high, and in order to spread as an individual level, it is essential that the cost is lower than the performance.

For example, in the design of a stepped-impedance low-pass filter (SI-LPF) which is one of the most essential MSL circuit, it is known that there is freedom in how to determine the characteristic impedance of low impedance line and the high impedance line. The larger their ratio, the better the agreement with the design theory. However, in practice the constraints determined by the dielectric constant and the thickness of the substrate cannot be ignored, and the freedom of choice is not very high except for high cost substrate and advanced manufacturing techniques or higher order filter synthesis techniques ${ }^{(7-9)}$. Furthermore, it is known that the actual cut-off frequency is lower than the design value, the specific adjustment method is not described in any literature. And no attempt has been made to improve properties using low cost substrates. So in this study, we examined the case where a low loss and high cost Teflon substrate is substituted with a low cost FR-4 substrate with reference to a commercially available microwave standard experiment kit. Good LPF characteristics are obtained by appropriately selecting the impedance ratio of the stepped-impedance (SI) line with use of the cutoff frequency adjustment. This paper is organized as follows. Section 2 describes the design method including line impedance determination method, single SI element characteristics, and cutoff frequency adjustment method. Section 3 explains the fabrication and measurement method. Section 4 discusses the measurement results and finally Section 5 summarizes the results. 


\section{Design Method}

\subsection{Design Theory}

Microstrip $L$ and $C$ elements can be expressed by gap / chip capacitors, loop / spiral inductors inserted in series ${ }^{(10)}$, open-ended or short-circuited resonators, and H-plane discontinuities that inserts short transmission lines with different line widths in series. In particular, the method using the discontinuous step of the transmission line is called a stepped impedance (SI) element and is similar to the inductive or capacitive windows known as waveguide $L$ and $C$ elements ${ }^{(11)}$. The design of the desired SI element is a little cumbersome, but it is an effective technique for reducing the overall circuit size compared to the method using a resonator. Following is the principle of the SI element design. The transmission line having the length $l$ shown in Fig. 1(a) can be represented by the T-type equivalent circuit shown in Fig. 1(b) using conversion of the $Z$ parameters and $A B C D$ parameters. Here, $Z_{1}, Z_{2}$, and $Z_{3}$ are given by Eq. (1) and (2) ${ }^{(12)}$.

$$
\begin{aligned}
& Z_{1}=Z_{2}=j Z_{0} \tan \frac{\beta l}{2}, \\
& Z_{3}=-j Z_{0} \frac{1}{\sin \beta l},
\end{aligned}
$$

If the transmission line physical length $l$ is short compared to the wavelength and the electrical length $\beta l<\pi / 2, Z_{1}$ and $Z_{2}$ in Eq. (1) are both inductive, then Eq. (3) is obtained by putting $Z_{1}=Z_{2}=j X / 2$.



(a)

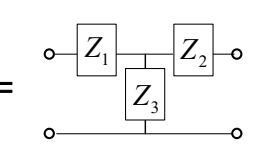

(b)

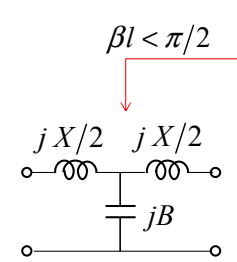

(c)

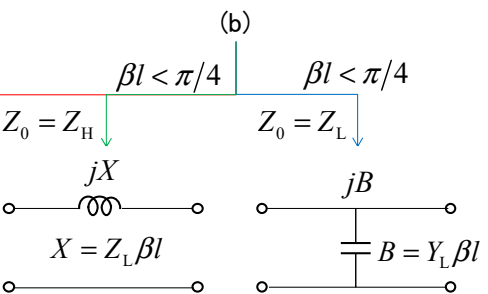

(d) (e)
Fig. 1. Equivalent circuit of the stepped impedance element. (a) Transmission line of electrical length is $\beta l$, (b) T-type equivalent circuit, (c) Equivalent circuit for $\beta l$ $<\pi / 2$, (d) Equivalent circuit for $\beta l<\pi / 4$ and $Z_{0}$ is large, (e) Equivalent circuit for $\beta l<\pi / 4$ and $Z_{0}$ is small.



Fig. 2. Lumped circuit model by LTspice. Cutoff frequency $f_{\mathrm{c}}=2.5 \mathrm{GHz}$, Butterworth filter of order $N=$ $6, C_{1}=0.659 \mathrm{pF}, L_{2}=4.502 \mathrm{nH}, C_{3}=2.460 \mathrm{pF}, L_{4}=$ $6.149 \mathrm{nH}, C_{5}=1.801 \mathrm{pF}, L_{6}=1.648 \mathrm{nH}$.

$$
\frac{X}{2}=Z_{0} \tan \frac{\beta l}{2},
$$

On the other hand, $Z_{3}$ in Eq. (2) is always negative pure imaginary, so it becomes capacitive. If $Y_{3}=1 / Z_{3}=j B$, Eq. (4) is obtained.

$$
B=Y_{0} \tan \beta l,
$$

In this case, the low-pass T-type equivalent circuit shown in Fig. 1(c) is obtained. When the electrical length is even shorter $\beta l<\pi / 4$, and $Z_{0}$ is sufficiently large, that is, when $Z_{0}$ $=Z_{\mathrm{H}}$, Eq. (3) can be approximated by Eq. (5). It becomes a series element of $L$ as shown in Fig. 1(d).

$$
X=Z_{L} \beta l,
$$

On the other hand, when $Z_{0}$ is sufficiently small, that is, when $Z_{0}=Z_{\mathrm{L}}$, Eq. (4) can be approximated by Eq. (6), resulting in the parallel element of $C$ as shown in Fig. 1(e).

$$
B=Y_{L} \beta l=\frac{\beta l}{Z_{L}},
$$

The electrical length is given by Eq. (7) and (8).

$$
\begin{gathered}
\beta l=\frac{R_{0} L_{k}}{Z_{H}}, \\
\beta l=\frac{Z_{l} C_{k}}{R_{0}},
\end{gathered}
$$

Where, $L_{k}$ and $C_{k}$ represent the low-pass prototype value $g_{k}$, $R_{0}$ is the filter impedance, and $\omega_{\mathrm{c}}$ is the cutoff angular frequency. If frequency scaling rule is applied, then the $L$ and $C$ element values $L^{\prime}{ }_{k}$ and $C^{\prime}{ }_{k}$ are given by Eq. (9) and (10).

$$
\begin{aligned}
& L_{k}^{\prime}=\frac{R_{0} L_{k}}{\omega_{c}}, \\
& C_{k}^{\prime}=\frac{C_{k}}{R_{0} \omega_{c}},
\end{aligned}
$$




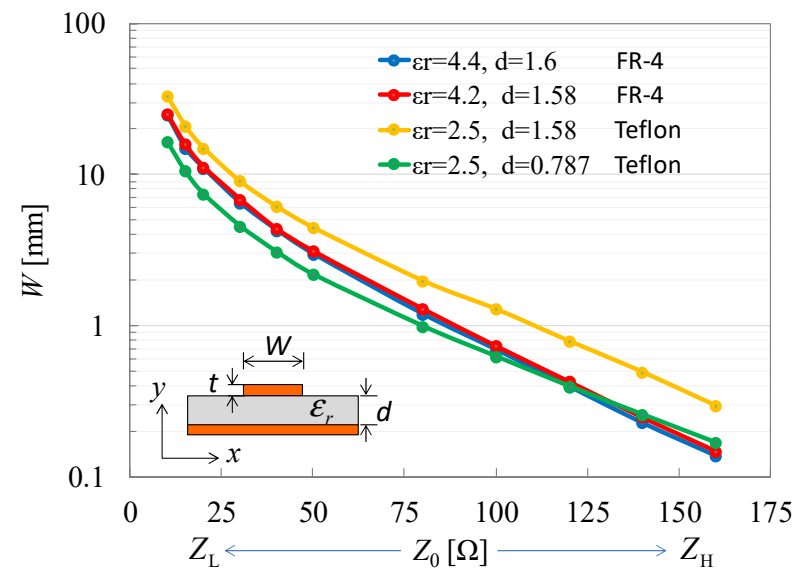

Fig. 3. Line width $W[\mathrm{~mm}]$ with respect to the characteristic impedance $Z_{0}[\Omega]$.

Assuming that the design specifications are the same as in the example, the cutoff frequency is $f_{\mathrm{c}}=2.5 \mathrm{GHz}$, the insertion loss is over $20 \mathrm{~dB}$ at $4 \mathrm{GHz}$, and the Butterworth filter of order $N=6$ are selected. Then, the LPF consisting of $L C$ circuit is found as shown in Fig. 2. In this study, the circuit simulator LTspice is used to derive the transmission characteristics.

\subsection{Line impedance selection}

Fig. 3 shows the variation of the microstrip line width $W$ with respect to the characteristic impedance $Z_{0}$. Four types of dielectric constant $\varepsilon_{\mathrm{r}}$ and substrate thickness $d$ are selected from available dielectric substrates ${ }^{(6)(12-13)}$. From the point of view of design and manufacturing, the line width of high impedance $Z_{\mathrm{H}}$ should be as large as possible because of the easy manufacturing, and the line width of low impedance $Z_{\mathrm{L}}$ should be as small as possible in order to suppress the $\lambda / 4$ resonance transverse to the transmission direction. Although these requirements are contrary to the nature of the MSL characteristic impedance, it is confirmed the smaller dielectric constant $\varepsilon_{\mathrm{r}}$ and the substrate thickness $d$ tend to be easier to design from Fig. 2. It is obvious that a thick FR-4 substrate is disadvantageous compared to a thin Teflon substrate though the manufacturing cost is $1 / 10$ of Teflon substrate. Because our motivation is to improve the low pass characteristics at low cost, a thick and high loss FR-4 substrate is used.

To verify the design theory, some $Z_{\mathrm{L}}$ and $Z_{\mathrm{H}}$ are examined by using the EM simulator HFSS v11.1 with the reference ${ }^{(12)}$. The substrate parameters are $\varepsilon_{\mathrm{r}}=4.2, \tan \delta=$ $0.02, d=1.58 \mathrm{~mm}, t=30 \mu \mathrm{m}$. The conductor is assumed as a perfect conductor. Fig. 4 shows the LPF characteristics for changes in $Z_{\mathrm{H}}$ where $Z_{\mathrm{L}}$ is fixed at $20 \Omega$. The lumped constant design theory by using the circuit simulator

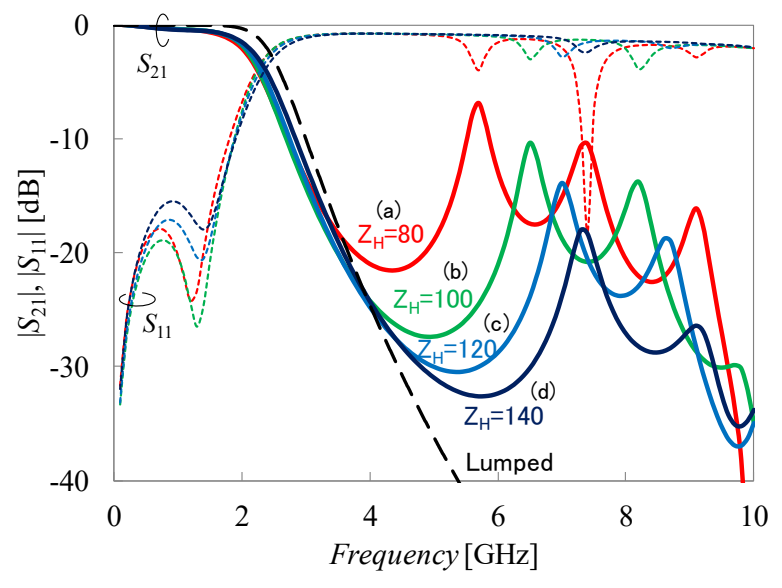

Fig. 4. LPF characteristics for changes in $Z_{\mathrm{H}}$. Where low impedance line is fixed at $Z_{\mathrm{L}}=20 \Omega$.

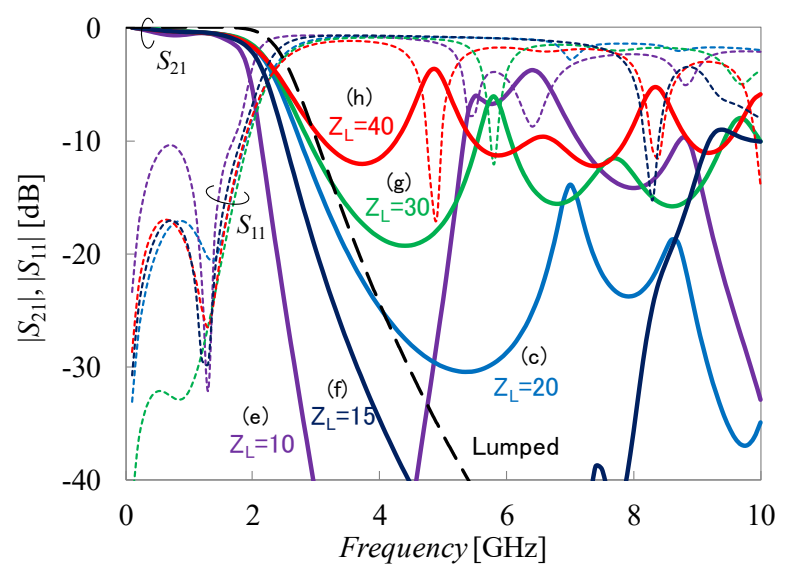

Fig. 5. LPF characteristics for changes in $Z_{\mathrm{L}}$. Where high impedance line is fixed at $Z_{\mathrm{H}}=120 \Omega$.


Fig. 6. Circuit patterns. $Z_{0}=50 \Omega$ line with a length of $20 \mathrm{~mm}$ is connected to the both input and output port. 
Table 1. Design parameters.

\begin{tabular}{|c|c|c|c|c|c|}
\hline & Substrate & $\boldsymbol{\varepsilon}_{\boldsymbol{r}}$ & $\boldsymbol{d} \mathbf{[ m m}]$ & $\boldsymbol{Z}_{\mathbf{H}}[\mathbf{\Omega}]$ & $\boldsymbol{Z}_{\mathbf{L}}[\mathbf{\Omega}]$ \\
\hline$[\mathbf{6}]$ & Teflon & 2.5 & 0.787 & 100 & 10 \\
\hline$[\mathbf{1 2}]$ & FR-4 & 4.2 & 1.58 & 120 & 20 \\
\hline Presented & FR-4 & 4.4 & 1.6 & 120 & 15 \\
\hline
\end{tabular}

LTspice is plotted by a broken line. It is observed that increasing the $Z_{\mathrm{H}}$ improves the attenuation level, but if it is too large, the line width becomes thinner. It leads to manufacturing difficulties. On the other hand, Fig. 5 shows the LPF characteristics for changes in $Z_{\mathrm{L}}$ where $Z_{\mathrm{H}}$ is fixed at $120 \Omega$. The smaller the $Z_{\mathrm{L}}$, the sharper the attenuation skirt characteristics, but if it is too small, it deviates from the lumped constant design theory. In particular, a large change appears in the skirt characteristics between $Z_{\mathrm{L}}=20 \Omega$ and $Z_{\mathrm{L}}=10 \Omega$, and it changes from low-pass characteristics to band-stop characteristics. This is considered to be caused by that $C$ sections start to work as $\lambda / 4$ open stubs. From

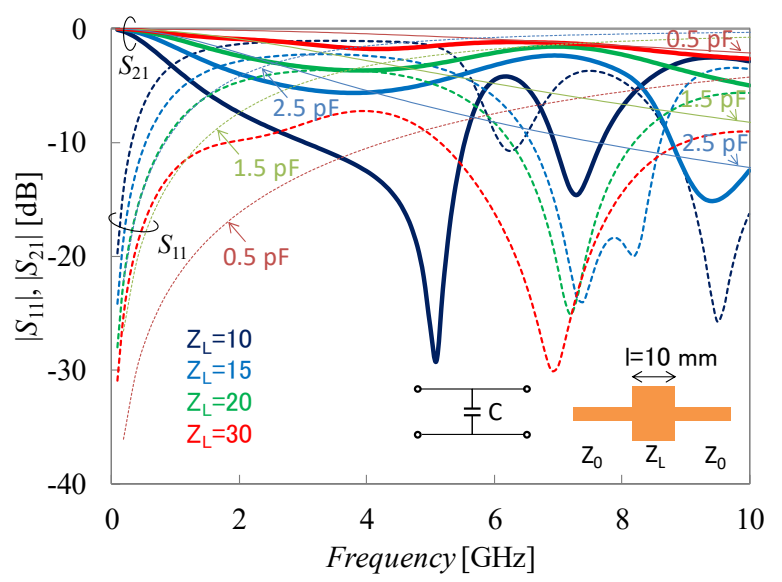

Fig. 7. Frequency characteristics for changes in $Z_{\mathrm{L}}$ having the length of $10 \mathrm{~mm}$ between lines of $Z_{0}=50 \Omega$.



Fig. 8. Frequency characteristics for changes in $Z_{\mathrm{L}}$ having the length of $5 \mathrm{~mm}$ between lines of $Z_{0}=50 \Omega$. these examinations, it is found that $Z_{\mathrm{H}}$ works to improve the attenuation level, and $Z_{\mathrm{L}}$ works to determine the slope of the attenuation curve. The circuit patterns in Fig. 4 and 5 are as shown in Fig. 6. If the $\lambda / 4$ transverse resonance generated in the line $Z_{\mathrm{L}}$ is used as the cutoff region of the LPF, the high frequency cutoff characteristics can be improved. Therefore, in this study, we adopts $Z_{\mathrm{L}}=15 \Omega$, which resonates transversally around $7 \mathrm{GHz}$, as the low impedance line. Table 1 shows the design parameters used for comparison. Design parameters in reference ${ }^{(12)}$ and this paper is similar, but the difference of the skirt characteristic is remarkable as shown in later.

\subsection{Characteristics of single SI element}

The reason why the selected values of $Z_{\mathrm{L}}$ and $Z_{\mathrm{H}}$ deviate from the LPF characteristics of the design theory is examined using an EM simulator, focusing on the characteristics of the $L$ and $C$ single-step elements. As

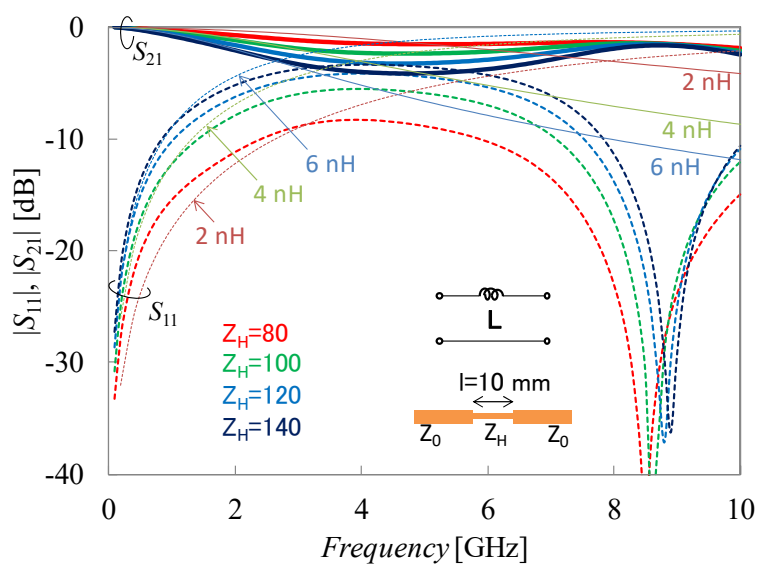

Fig. 9. Frequency characteristics for changes in $Z_{\mathrm{H}}$ having the length of $10 \mathrm{~mm}$ between lines of $Z_{0}=50 \Omega$.

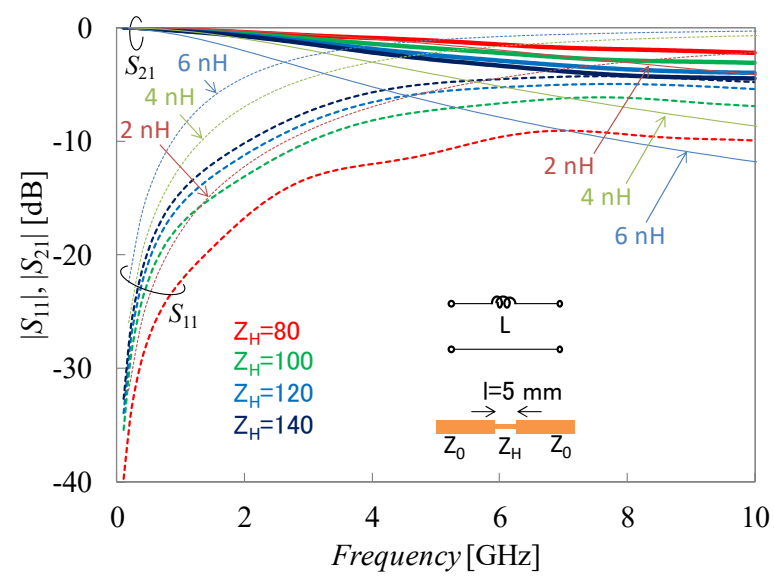

Fig. 10. Frequency characteristics for changes in $Z_{\mathrm{H}}$ having the length of $5 \mathrm{~mm}$ between lines of $Z_{0}=50 \Omega$. 
shown in Fig. 2, the lumped element values required for the design are $0.5-2.5 \mathrm{pF}$ in the $C$ section and about 2-6 $\mathrm{nH}$ in the $L$ section. Fig. 7 and 8 show the frequency characteristics of a single $Z_{\mathrm{L}}$ section. Two different section lengths, $l=10 \mathrm{~mm}$ and $l=5 \mathrm{~mm}$, are examined. For comparison, the $\left|S_{21}\right|$ and $\left|S_{11}\right|$ characteristics of the theoretical lumped element $C$ parallel circuit are shown. The theoretical reflection and transmission characteristics of a shunt $C$ element can be calculated by equations (11) and (12). Where $Z_{0}=1 / Y_{0}$ is the characteristic impedance of the input / output lines.

$$
\begin{aligned}
& \left|S_{11}\right|=\frac{\omega C}{\sqrt{4 Y_{0}^{2}+(\omega C)^{2}}}, \\
& \left|S_{21}\right|=\frac{2 Y_{0}}{\sqrt{4 Y_{0}^{2}+(\omega C)^{2}}},
\end{aligned}
$$

Similarly, the theoretical reflection and transmission characteristics of a series $L$ element are given by equations (13) and (14).

$$
\begin{aligned}
& \left|S_{11}\right|=\frac{\omega L}{\sqrt{4 Z_{0}^{2}+(\omega L)^{2}}}, \\
& \left|S_{21}\right|=\frac{2 Z_{0}}{\sqrt{4 Z_{0}^{2}+(\omega L)^{2}}},
\end{aligned}
$$

When $l=10 \mathrm{~mm}$ in Fig. 7, $C$ value larger than $2.5 \mathrm{pF}$ can be expressed on the low frequency, but the high frequency characteristics deteriorate due to the influence of the $\lambda / 2$ passband resonance point near $7 \mathrm{GHz}$. However, the high-frequency characteristics are improved at $l=5 \mathrm{~mm}$ in Fig. 8, and it is a good approximation except for the $\lambda / 4$ resonance point that operates as a stub with $Z_{\mathrm{L}}=10 \Omega$ and $Z_{\mathrm{L}}=15 \Omega$. However, the $\lambda / 4$ resonance near $8 \mathrm{GHz}$ with $Z_{\mathrm{L}}$ $=15$ can be used to reinforce the LPF stopband characteristics.

On the other hand, in the $L$ section, the required inductances about 2-6 $\mathrm{nH}$ are obtained on the low frequency in Fig. 9, but on the high frequency, the frequency characteristics deteriorate due to the influence of the $\lambda / 2$ passband resonance near $9 \mathrm{GHz}$. This is improved in the case of $l=5 \mathrm{~mm}$ in Fig. 10, but the required inductance value is not obtained. In both cases, if the section length $l$ is increased, larger $L$ and $C$ element values can be obtained. However, since the passband resonance point appears, the spurious characteristics in the high frequency deteriorate. In summary, the desired $L$ can be obtained by increasing $l$, but the spurious response after $5 \mathrm{GHz}$ deteriorates. If $l$ is

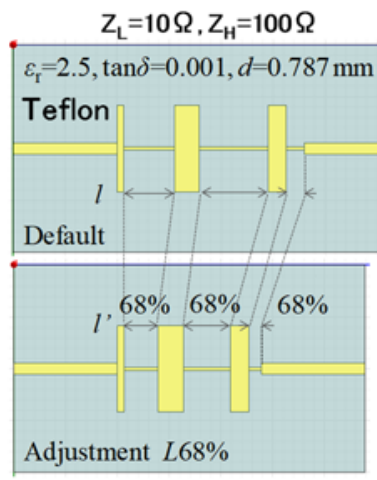

(a)



(b)
Fig. 11. Circuit pattern before and after $L$ section adjustment. (a) Teflon substrate with $\varepsilon_{\mathrm{r}}=2.5, d=0.787$ $\mathrm{mm}$, (b) FR-4 substrate with $\varepsilon_{\mathrm{r}}=4.4, d=1.6 \mathrm{~mm}$. The upper sides are the pattern that applied the design theory, and the lower sides are the pattern where the $L$ section is shortened to adjust the cutoff frequency.

shortened, the desired $L$ cannot be obtained, but the spurious response is improved. Since these are in a trade-off relationship, it is necessary to find and design a compromise.

\subsection{Cutoff Frequency Adjustment}

According to the design theory, the cutoff frequency $f_{\mathrm{c}}$ appears slightly lower than the design value as shown in Fig. 4 and Fig. 5. The fact that the cutoff frequency $f_{\mathrm{c}}$ is smaller than the designed value means that $C$ or $L$ represented by SI elements appears larger than the designed value. As a physical reason for this phenomenon, the fringing effect of $C$ section and the skin effect of $L$ section are considered. Therefore, it is considered that the method of reducing $C$ is proper for the frequency adjustment, but there is no problem with the method of shortening $L$ if the

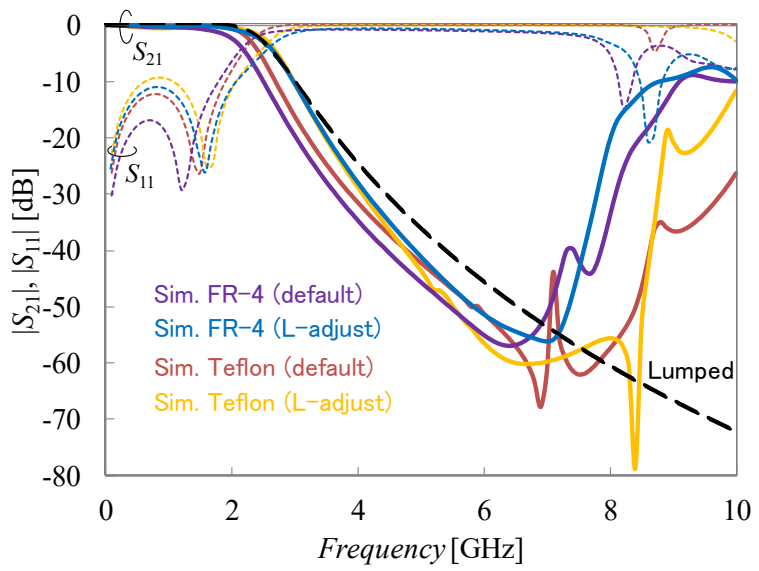

Fig. 12. Comparison of frequency characteristics before and after frequency adjustment. 
purpose is to raise $f_{\mathrm{c}}$. Though several experimental formulas have been introduced for the adjustment method of $\operatorname{MSL}^{(14)(15)}$, only the lengths of the $L$ section are shortened in the commercially available microwave experiment $\mathrm{kit}^{(9)}$. So in this study, the frequency adjustment is conducted similarly by shortening only $L$ sections. Fig. 11 shows the circuit pattern before and after the frequency adjustment. The upper side shows the circuit pattern to which the design theory ${ }^{(12)}$ is applied, and the lower side shows the pattern in which the $L$ section is shortened to adjust the cutoff frequency. Since only the $L$ section is reduced to about $68 \%$

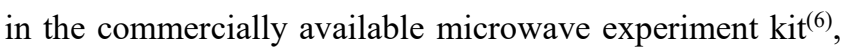
the FR-4 self-made model is reduced to $70 \%$ only in the $L$ section. The measured cutoff frequency before adjustment is $2.1 \mathrm{GHz}$ which is 0.84 times of the design frequency 2.5 GHz. If this is applied to the cutoff frequency formula of Eq. (15), the cutoff frequency can be increased to 1.2 times, which is the reciprocal of 0.84 , if $L$ section is shortened by 0.7 times. So, it can be seen that this adjustment rate is theoretically valid.

$$
f_{\mathrm{c}}=\frac{1}{2 \pi \sqrt{L C}},
$$

Fig. 12 shows the frequency characteristics before and after $L$ adjustment by the EM simulator. The circuit patterns are shown in Fig. 11. Thick solid lines indicate $\left|S_{21}\right|$ and thin dashed lines indicate $\left|S_{11}\right|$, and the five types of data show EM simulation values before frequency adjustment and after adjustment, lumped element circuit model design values. It can be seen that the cut-off frequency is improved by adjusting the $L$ section. It is also found that the cutoff frequency can be improved in the same way using the help of the EM simulator even if only $C$ is reduced to $60 \%$ or both $L$ and $C$ are reduced to $90 \%$ and $70 \%$, respectively. Adjusting $C$ is not easy due to the fringing effect. Adjustment requires an EM simulator.

\section{Fabrication and measurement method}

The image of a measurement sample is shown in Fig. 13 lower. And an image of the commercial experimental kit MW-1000 is also shown upper for comparison. Physical dimensions are as shown in Table 2 and Table 3. In this table, $N$ is the filter order, $g_{k}$ is the filter prototype value, $W$ is the line width, $l$ is the line length before adjustment, and $l$ ' means after adjustment. Pattern fabrication is performed by the laboratory self-etching system ${ }^{(16)}$ using a glass fiber base epoxy resin copper clad laminate NZ-G30KR made by

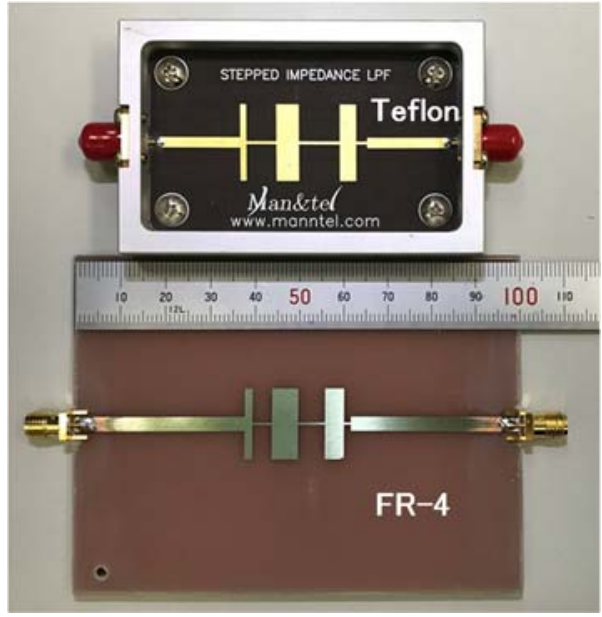

Fig. 13. Measurement circuit samples. Commercial microwave experiment kit MW-1000 using Teflon substrate (upper), FR-4 substrate (lower).

Table 2. Physical dimensions of MW-1000.

\begin{tabular}{|c|c|c|c|c|c|c|}
\hline $\boldsymbol{N}$ & $\boldsymbol{g}_{\boldsymbol{k}}$ & $\boldsymbol{Z}_{\mathbf{L}, \boldsymbol{Z}_{\mathbf{H}}[\boldsymbol{\Omega}]}$ & $\boldsymbol{\beta l}[\mathbf{d e g}]$ & $\boldsymbol{W}[\mathbf{m m}]$ & $\boldsymbol{l}[\mathbf{m m}]$ & $\boldsymbol{l}^{\prime}[\mathbf{m m}]$ \\
\hline 1 & 0.5176 & 10 & 5.9 & 16.7 & 1.29 & 1.31 \\
\hline 2 & 1.4142 & 100 & 40.5 & 0.63 & 9.70 & 6.58 \\
\hline 3 & 1.9318 & 10 & 22.1 & 16.7 & 4.81 & 4.90 \\
\hline 4 & 1.9318 & 100 & 55.3 & 0.63 & 13.2 & 9.02 \\
\hline 5 & 1.4142 & 10 & 16.2 & 16.7 & 3.52 & 3.59 \\
\hline 6 & 0.5176 & 100 & 14.8 & 0.63 & 3.55 & 2.42 \\
\hline
\end{tabular}

Table 3. Physical dimensions of FR-4 sample.

\begin{tabular}{|c|c|c|c|c|c|c|}
\hline $\boldsymbol{N}$ & $\boldsymbol{g}_{\boldsymbol{k}}$ & $\boldsymbol{Z}_{\mathbf{L}}, \boldsymbol{Z}_{\mathbf{H}}[\boldsymbol{\Omega}]$ & $\boldsymbol{\beta} \boldsymbol{l}[\mathbf{d e g}]$ & $\boldsymbol{W}[\mathbf{m m}]$ & $\boldsymbol{l}[\mathbf{m m}]$ & $\boldsymbol{l}^{\prime}[\mathbf{m m}]$ \\
\hline 1 & 0.5176 & 15 & 8.9 & 15.7 & 1.51 & 1.51 \\
\hline 2 & 1.4142 & 120 & 33.8 & 0.41 & 6.55 & 4.59 \\
\hline 3 & 1.9318 & 15 & 33.2 & 15.7 & 5.64 & 5.64 \\
\hline 4 & 1.9318 & 120 & 46.1 & 0.41 & 8.95 & 6.27 \\
\hline 5 & 1.4142 & 15 & 24.3 & 15.7 & 4.13 & 4.13 \\
\hline 6 & 0.5176 & 120 & 12.4 & 0.41 & 2.40 & 1.68 \\
\hline
\end{tabular}

Sunhayato ${ }^{(13)}$. The measuring instrument used is a vector network analyzer (VNA) Agilent E8362C. Coaxial SMA connectors RS 526-5785 are attached to both ends of the substrate for connection to the coaxial calibration surface. The tip of the $3.5 \mathrm{~mm}$ coaxial connector is set as the calibration plane, then the measurement sample is inserted into the calibration surface, and $S_{21}$ and $S_{11}$ are measured in the range of 0.01-10 GHz.

\section{Results and Discussion}

The measurement results are shown in Fig. 14. Thick solid lines indicate $\left|S_{21}\right|$ and thin dashed lines indicate $\left|S_{11}\right|$, and the five types of data show measured value, EM simulation, lumped element circuit model design values, EM simulation of FR-4 in the case of $Z_{\mathrm{L}}=20 \Omega$ and $Z_{\mathrm{H}}=120$ $\Omega$ used for comparison, and measured value of Teflon 


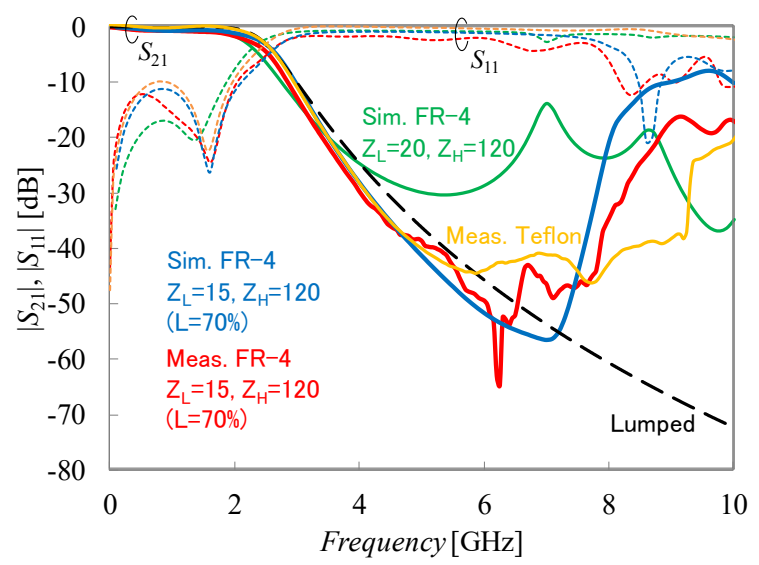

Fig. 14. Measurement results.

substrate MW-1000 used for comparison. When two types of EM simulation are compared, sufficient attenuation characteristics are not obtained in the case of $Z_{\mathrm{L}}=20 \Omega$. However, in this study where $Z_{\mathrm{L}}=15 \Omega$, it can be seen that attenuation exceeding the measured reference value of Teflon substrate is obtained by the $\lambda / 4$ transverse resonance technique near $7 \mathrm{GHz}$. In addition, the measured value in this study agree well with the EM simulation up to around 6 $\mathrm{GHz}$, and the design specifications are fully satisfied up to $7 \mathrm{GHz}$. The difference between the measured value and the simulation above $6 \mathrm{GHz}$ is probably due to the skin effect which is not calculated accurately in the simulation because of the coarse mesh in FEM algorithm. Although, the high frequency spurious response is worse than the commercial reference model, the effectiveness of the presented design method using the $\lambda / 4$ transverse resonance technique at cutoff region and the validity of the frequency adjustment method are confirmed.

\section{Conclusions}

A series of process from low cost design to fabrication of a SI-LPF which is one of the most essential MSL circuit is investigated by using a low cost FR-4 substrate instead of a low loss and high cost Teflon substrate. By appropriately selecting the impedance ratio of the SI line with use of the $\lambda / 4$ transverse resonance technique at cutoff region, good LPF characteristics are obtained as in the commercial microwave experiment kit using a high cost Teflon substrate. So the manufacturing cost is reduced to about one tenth. Then, frequency adjustment is performed with reference to a commercial microwave experimental kit in addition to the standard design theory, and comparisons are made between the design values by the circuit simulator, the EM simulation and the measurement. It is confirmed that the frequency characteristics of the manufactured sample agree well up to $6 \mathrm{GHz}$. In the future, we plan to study the manufacturing accuracy of the high impedance line, the influence of the skin effect and the combination with an auxiliary circuit to improve the high frequency spurious characteristics.

\section{References}

(1) R. E. Collin : "Foundations for Microwave Engineering - 2nd edition", Wiley-IEEE Press, 2001

(2) R. F. Harrington : "Time-Harmonic Electromagnetic Fields 2nd Edition", Wiley-IEEE Press, 2001

(3) SPC : "Waveguide microwave experiment", Available online: www.spc.co.jp/products/microwave (accessed on 17 April 2019)

(4) DreamCatcher Asia : "Complete resources for lecturers" Available online: www.dreamcatcher.asia/cw (accessed on 17 April 2019)

(5) FESTO : "LabVolt Series", Available online: www.labvolt.com/solutions (accessed on 29 November 2019)

(6) Man \& tel Co., Ltd. : "MW-1000", Available online: www.pid-control.com (accessed on 29 November 2019)

(7) S. Arakelyan, H. Lee, D. Han, A. Babajanyan, G. Berthiau, B. Friedman, K. Lee, "Microwave Heating Visualization for Carbon Fibers Composite Material: Development of Tunable Microstrip Structures", IEEE Trans. MTT. Vol. 66, No. 2, February 2018.

(8) C. Chen, C. Sung, Y. Su, "A Multi-Stub Lowpass Filter", IEEE Microwave and Wireless Component Letters, Vol. 25, No. 8, August 2015

(9) A. Casanueva, A. Leon, O. Gonzalez, A. Mediavilla, "A compact microstrip step-impedance low-pass filter (SILPF) using complementary split ring resonators (CSRRs) ”, 2009 IEEE MTT-S International Microwave Workshop on Wireless Sensing, Local Positioning, and RFID

(10)D. M. Pozar : "Microwave Engineering - 3rd edition", pp.227-228, Wiley, 2005

(11)N. Marcuvitz : "Waveguide handbook", pp.217-229, IEE, 1993

(12)D. M. Pozar : "Microwave Engineering - 3rd edition", pp.412-416, Wiley, 2005

(13) Sunhayato : "NZ-G30KR", Available online: 
www.sunhayato.co.jp (accessed on 29 November 2019)

(14) S. W. Wedge, R. Compton, D. Rutledge : "PUFF, Computer Aided Design for Microwave Integrated Circuits", pp.27-29, 1997

(15) T. H. Lee : "Planar Microwave Engineering", pp.175-176, Cambridge, 2004

(16) Y. Kusama : "Circuit board etching procedure manual", Available online: www.kusamalab.org/standard/measure/etching.pdf (accessed on 18 April 2019) 